\title{
A Note on Cubic Convolution Interpolation
}

\author{
Erik Meijering and Michael Unser
}

IEEE Transactions on Image Processing, vol. 12, no. 4, April 2003, pp. 477-479

\begin{abstract}
We establish a link between classical osculatory interpolation and modern convolutionbased interpolation and use it to show that two well-known cubic convolution schemes are formally equivalent to two osculatory interpolation schemes proposed in the actuarial literature about a century ago. We also discuss computational differences and give examples of other cubic interpolation schemes not previously studied in signal and image processing.
\end{abstract}

\section{Introduction}

Polynomial interpolation methods have been studied quite extensively in the signal and image processing literature of the past three decades $[7,8,14]$. An example of such methods is Lagrange central interpolation of given, fixed degree, which is known to yield interpolants that are not continuously differentiable $[10,11]$. In order to obtain smoother interpolants, as may be required for some applications, several alternative interpolation methods have been proposed. Popular examples of these are the so-called cubic convolution interpolation methods, of which the ones proposed by Keys [5] are the most well known.

It is probably less well known that methods for obtaining smooth interpolants have been developed in other areas of applied mathematics since the second half of the nineteenth century. In this brief note we establish a link between classical osculatory interpolation and modern convolution-based interpolation and use it to show that both of Keys' cubic convolution schemes are formally equivalent to particular osculatory interpolation schemes proposed around the beginning of the twentieth century. We also discuss their computational differences and give explicit forms of the kernels that follow from other cubic osculatory interpolation schemes.

\section{Convolution-Based Interpolation}

Convolution-based interpolation of uniformly sampled data implies the use of an interpolation kernel $\varphi: \mathbb{R} \rightarrow \mathbb{R}$, which determines the weights to be assigned to the samples $f_{k}=f(k T)$ of an original function $f: \mathbb{R} \rightarrow \mathbb{R}$ in computing the value of the interpolant $\tilde{f}$ at any arbitrary $x \in \mathbb{R}$. For ease of notation, but without loss of generality, we will use $T=1$ in the remainder of this paper. In that case, the process may be described mathematically as

$$
\tilde{f}(x)=\sum_{k \in \mathbb{Z}} f_{k} \varphi(x-k) .
$$

As can readily be observed from this equation, it is necessary that in order for $\tilde{f}$ to be an interpolant, the kernel $\varphi$ must satisfy the criteria $\varphi(0)=1$ and $\varphi(k)=0, \forall k \neq 0$. A well-known example of such a kernel is the theoretically ideal, but computationally very 
unattractive sinc function. Other examples are the computationally very attractive, but theoretically far from ideal nearest-neighbor and linear interpolation kernel.

A considerably better trade-off between computational cost and accuracy is provided by the family of cubic convolution kernels, an example of which was used first in 1973 by Rifman [9]. These kernels consist of piecewise third-degree polynomials and are once continuously differentiable. Of special interest are the kernels proposed in 1981 by Keys [5], since they generally yield more accurate results than other kernels of the family. The first has an approximation order of $L=3$, which implies that the resulting interpolant converges to the original function as fast as the third power of the intersample distance. It also implies that the kernel is capable of reproducing polynomials up to second degree. This so-called third-order cubic convolution kernel - in computer graphics also known as the Catmull-Rom spline [1] — is defined as

$$
\varphi_{\mathrm{CC} 3}(x)= \begin{cases}\frac{3}{2}|x|^{3}-\frac{5}{2}|x|^{2}+1 & \text { if } 0 \leqslant|x| \leqslant 1, \\ -\frac{1}{2}|x|^{3}+\frac{5}{2}|x|^{2}-4|x|+2 & \text { if } 1 \leqslant|x| \leqslant 2, \\ 0 & \text { if } 2 \leqslant|x| .\end{cases}
$$

By extending the support of the kernel, while keeping the highest degree of the polynomial pieces to $n=3$, Keys [5] also obtained a cubic convolution kernel with order of approximation $L=4$. This so-called fourth-order cubic convolution kernel is defined as

$$
\varphi_{\mathrm{CC} 4}(x)= \begin{cases}\frac{4}{3}|x|^{3}-\frac{7}{3}|x|^{2}+1 & \text { if } 0 \leqslant|x| \leqslant 1, \\ -\frac{7}{12}|x|^{3}+3|x|^{2}-\frac{59}{12}|x|+\frac{5}{2} & \text { if } 1 \leqslant|x| \leqslant 2, \\ \frac{1}{12}|x|^{3}-\frac{2}{3}|x|^{2}+\frac{7}{4}|x|-\frac{3}{2} & \text { if } 2 \leqslant|x| \leqslant 3, \\ 0 & \text { if } 3 \leqslant|x| .\end{cases}
$$

\section{Osculatory Interpolation}

Osculatory interpolation has been described as that form of interpolation in which one employs in a sequence of interpolation intervals a corresponding sequence of interpolation curves forming a composite curve which, together with a specified number of its derivatives, is continuous throughout the range of interpolation [2]. Such interpolation schemes have been developed since the second half of the nineteenth century, primarily in the actuarial literature, and an overview of many of them was given as early as 1944 by Greville [2].

A convenient form in which to express osculatory interpolation formulae is the socalled Everett form, since it involves only the even-order central differences of the two given samples determining the interval in which to interpolate:

$$
\tilde{f}(x)=\tilde{f}(k+\xi)=F(\xi, \delta) f_{k+1}+F(1-\xi, \delta) f_{k},
$$

with $k=\lfloor x\rfloor, 0 \leqslant \xi \leqslant 1$, and $F(x, \delta)=\sum_{i=0}^{i_{\max }} F_{i}(x) \delta^{2 i}$ for some $i_{\max }$, where the $F_{i}$ are suitably chosen polynomial functions in $x$ such that the resulting interpolant satisfies prespecified criteria concerning its order of approximation and smoothness in terms of continuous differentiability. Here, the $p$ th-order central difference $\delta^{p}$ of any function $g$ is defined as $\delta^{p} g(x)=\delta^{p-1} g\left(x+\frac{1}{2}\right)-\delta^{p-1} g\left(x-\frac{1}{2}\right)$, with $\delta g(x)=g\left(x+\frac{1}{2}\right)-g\left(x-\frac{1}{2}\right)$.

An example of an osculatory interpolation formula is the one described by Karup [4] in 1899 and independently by King [6] in 1907, which is obtained from (4) by taking [2,13]

$$
F(x, \delta)=F_{\mathrm{KK}}(x, \delta)=x+\frac{1}{2} x^{2}(x-1) \delta^{2} .
$$


Similar to third-order cubic convolution, it yields a continuously differentiable third-degree piecewise polynomial interpolant and is capable of reproducing polynomials up to second degree. A second example is the formula proposed by Henderson [3] in 1906, which is obtained from (4) by taking [2]

$$
F(x, \delta)=F_{\mathrm{H}}(x, \delta)=x+\frac{1}{6} x\left(x^{2}-1\right) \delta^{2}-\frac{1}{12} x^{2}(x-1) \delta^{4} .
$$

Similar to fourth-order cubic convolution, this scheme yields a continuously differentiable third-degree piecewise polynomial interpolant and is capable of reproducing polynomials up to third degree.

\section{Establishing the Link}

In his 1946 landmark paper [12] on the approximation of equidistant data by analytic functions, in which he introduced the special type of osculatory interpolation known as spline interpolation, Schoenberg also studied previously given classical interpolation schemes and pointed out that these interpolation schemes too may be written in the form (1), where the hidden kernel $h$ reveals itself as the response to the discrete impulse function defined by $f_{0}=1$ and $f_{k}=0, \forall k \neq 0$. Using this approach, he obtained the Lagrange central interpolation kernels and also the kernel involved in an osculatory interpolation scheme due to Jenkins $[2,12]$.

By proceeding in a similar fashion, we may obtain a general expression for the hidden kernels of osculatory interpolation schemes. To this end we use the expansion

$$
\delta^{2 i} f_{k}=\sum_{m=0}^{2 i}\left(\begin{array}{l}
2 i \\
m
\end{array}\right)(-1)^{m} f_{k-m+i}
$$

which holds for all $i \geqslant 0$ integer. Substituting this expansion into (4) and using the general expression for $F(x, \delta)$, we obtain

$$
\tilde{f}(x)=\tilde{f}(k+\xi)=\sum_{i=0}^{i_{\max }} \sum_{m=0}^{2 i}\left(\begin{array}{l}
2 i \\
m
\end{array}\right)(-1)^{m}\left(F_{i}(\xi) f_{k-m+i+1}+F_{i}(1-\xi) f_{k-m+i}\right)
$$

Substituting $\xi=x-k=\beta^{1}(1-x+k)$ and $1-\xi=1-x+k=\beta^{1}(x-k)$, where $\beta^{1}(x)$ is the linear interpolation kernel, or first-degree B-spline $[8,12,14]$, and using the facts that $\beta^{1}(-x)=\beta^{1}(x), \forall x \in \mathbb{R}, \beta^{1}(x)=0, \forall|x| \geqslant 1$, and $F_{i}(0)=0, \forall i$, we find that the two terms between square brackets in (8) may be combined to $\sum_{l \in \mathbb{Z}} F_{i}\left(\beta^{1}(x-k-l)\right) f_{k-m+i+l}$, so that we obtain the following expression for the impulse response, i.e., the kernel:

$$
\varphi(x)=\sum_{i=0}^{i_{\max }} \sum_{m=0}^{2 i}\left(\begin{array}{l}
2 i \\
m
\end{array}\right)(-1)^{m} F_{i}\left(\beta^{1}(x-m+i)\right) .
$$

By taking $i_{\max }=1, F_{0}(x)=x$, and $F_{1}(x)=\frac{1}{2} x^{2}(x-1)$, it now easily follows from (9) that the kernel involved in the Karup-King type of osculatory interpolation is precisely (2). Similarly, taking $i_{\max }=2, F_{0}(x)=x, F_{1}(x)=\frac{1}{6} x\left(x^{2}-1\right)$, and $F_{2}(x)=-\frac{1}{12} x^{2}(x-1)$, we find that the kernel involved in Henderson's type of osculatory interpolation is precisely (3). 


\section{Discussion}

From our analysis in the previous section it follows that Karup-King osculatory interpolation is formally equivalent to Keys third-order cubic convolution interpolation. Since the third-order cubic convolution kernel defined by Keys is a special case of an infinitely large family of cubic interpolation kernels having the same properties in terms of approximation order and regularity, this is a rather surprising result. The same can be said about the equivalence of Henderson osculatory interpolation and Keys fourth-order cubic convolution interpolation.

Notwithstanding their formal equivalence, however, it will be clear that the schemes are rather different from a computational perspective. Comparison of (1) and (2) versus (4) and (5), for example, shows that for a single interpolation, Keys' third-order cubic convolution scheme requires the evaluation of four cubic polynomials, compared to two cubic and two linear polynomials in the case of Karup-King osculatory interpolation. Working out the details, it follows that for a separable interpolation operation the former scheme requires a minimum of 23 floating-point operations (flops) per pixel per dimension, whereas the latter requires only 13. In the more complex case of Keys' fourth-order cubic convolution scheme and Henderson's osculatory interpolation scheme it follows that the number of flops is 37 for the former versus 30 for the latter. In the more general case of nonseparable operations, the gain can be made even higher if the central difference values are precomputed. This, however, requires more computer memory. It appears therefore that the osculatory equivalents of convolution-based interpolation schemes allow for faster though more memory demanding algorithms.

Furthermore we note that the schemes of Karup-King and Henderson are but two examples of the numerous osculatory interpolation schemes discussed by Greville [2]. Although a full-fledged study of these schemes is outside the scope of the present correspondence, it may be worthwhile to give explicit forms and properties of other interesting cubic interpolation kernels that follow from them. To the best of our knowledge, these kernels have not been investigated before in the signal and image processing literature.

As a first example we mention the scheme - apparently also due to Henderson - given by $F_{0}(x)=x$ and $F_{1}(x)=-6 F_{2}(x)=\frac{1}{6} x\left(x^{2}-1\right)$. Applying (9) we find that its kernel is

$$
\varphi(x)= \begin{cases}\frac{7}{9}|x|^{3}-\frac{3}{2}|x|^{2}-\frac{5}{18}|x|+1 & \text { if } 0 \leqslant|x| \leqslant 1 \\ -\frac{11}{36}|x|^{3}+\frac{7}{4}|x|^{2}-\frac{28}{9}|x|+\frac{5}{3} & \text { if } 1 \leqslant|x| \leqslant 2 \\ \frac{1}{36}|x|^{3}-\frac{1}{4}|x|^{2}+\frac{13}{18}|x|-\frac{2}{3} & \text { if } 2 \leqslant|x| \leqslant 3 \\ 0 & \text { if } 3 \leqslant|x|\end{cases}
$$

This kernel shares the property with the Keys-Henderson fourth-order kernel (3) that its support is $[-3,3]$ and its order of approximation $L=4$. Its regularity, however, is only $C^{0}$, similar to the cubic Lagrange central interpolation kernel $[10,11]$.

A second scheme mentioned by Greville is given by $F_{0}(x)=x, F_{1}(x)=x(x-1)((2 \alpha+$ $\left.\left.\frac{1}{2}\right) x-\alpha\right)$, and $F_{2}(x)=\frac{1}{2} \alpha x^{2}(x-1)$. Because of its free parameter, $\alpha$, it constitutes a whole family of cubic interpolation kernels, the general form of which follows from (9) as

$$
\varphi(x)= \begin{cases}\left(\alpha+\frac{3}{2}\right)|x|^{3}-\left(\alpha+\frac{5}{2}\right)|x|^{2}+1 & \text { if } 0 \leqslant|x| \leqslant 1, \\ \frac{1}{2}(\alpha-1)|x|^{3}-\left(3 \alpha-\frac{5}{2}\right)|x|^{2}+\left(\frac{11}{2} \alpha-4\right)|x|-(3 \alpha-2) & \text { if } 1 \leqslant|x| \leqslant 2, \\ -\frac{1}{2} \alpha|x|^{3}+4 \alpha|x|^{2}-\frac{21}{2} \alpha|x|+9 \alpha & \text { if } 2 \leqslant|x| \leqslant 3, \\ 0 & \text { if } 3 \leqslant|x| .\end{cases}
$$


Analyzing (11) we observe that the family includes both Keys' third-order kernel (2) and his fourth-order kernel (3), respectively corresponding to $\alpha=0$ and $\alpha=-\frac{1}{6}$. For any $\alpha \in \mathbb{R}$, the resulting kernel has at least regularity $C^{1}$ and approximation order $L=3$.

Finally we mention the even more general, two-parameter scheme given by $F_{0}(x)=x$, $F_{1}(x)=x(x-1)\left(\left(2 \alpha+\frac{1}{2}\right) x-\alpha\right), F_{2}(x)=x\left(\left(\frac{1}{2} \alpha+2 \beta\right) x^{2}-\left(\frac{1}{2} \alpha+3 \beta\right) x+\beta\right)$, and $F_{3}(x)=\frac{1}{2} \beta x^{2}(x-1)$. The general form of the family of kernels following from this scheme is

$$
\varphi(x)= \begin{cases}\left(\alpha-\frac{5}{2} \beta+\frac{3}{2}\right)|x|^{3}-\left(\alpha-\frac{5}{2} \beta+\frac{5}{2}\right)|x|^{2}+1 & \text { if } 0 \leqslant|x| \leqslant 1, \\ \frac{1}{2}(\alpha-\beta-1)|x|^{3}-\left(3 \alpha-\frac{9}{2} \beta-\frac{5}{2}\right)|x|^{2}+\left(\frac{11}{2} \alpha-10 \beta-4\right)|x|-(3 \alpha-6 \beta-2) & \text { if } 1 \leqslant|x| \leqslant 2, \\ -\frac{1}{2}(\alpha-3 \beta)|x|^{3}+\left(4 \alpha-\frac{25}{2} \beta\right)|x|^{2}-\left(\frac{21}{2} \alpha-34 \beta\right)|x|+(9 \alpha-30 \beta) & \text { if } 2 \leqslant|x| \leqslant 3, \\ -\frac{1}{2} \beta|x|^{3}+\frac{11}{2} \beta|x|^{2}-20 \beta|x|+24 \beta & \text { if } 3 \leqslant|x| \leqslant 4, \\ 0 & \text { if } 4 \leqslant|x| .\end{cases}
$$

Similar to the previously mentioned kernel, (11), which corresponds to the special case $\beta=0$, this kernel has at least regularity $C^{1}$ and approximation order $L=3$.

\section{Conclusions}

In this correspondence we have derived a general expression for the kernels implicitly involved in classical osculatory interpolation schemes. Using this formula we have shown that the still popular cubic convolution kernels described by Keys [5] twenty years ago are precisely the kernels involved in the osculatory interpolation schemes proposed by Karup and King [4,6] and Henderson [3] around 1900. We have also discussed their computational differences, from which we conclude that the osculatory versions are computationally cheaper, but require additional memory. Finally we have given the explicit forms and properties of other cubic convolution interpolation kernels implicitly used in the actuarial literature for a long time now, but which to the best of our knowledge have not been investigated before in the context of signal and image processing. Further study will be required to reveal the suitability of these kernels and the optimal values of their free parameters for specific applications.

\section{Acknowledgments}

The work described in this paper was supported in part by the Netherlands Organization for Scientific Research (NWO). The original version of the paper was written while the first author was with the Biomedical Imaging Group (BIG) of the Swiss Federal Institute of Technology in Lausanne (EPFL), Switzerland, and the revision was completed while he was with the Biomedical Imaging Group Rotterdam (BIGR) of the Erasmus MC-University Medical Center Rotterdam, the Netherlands. The authors are grateful to Dr. Erik Jan Dubbink (Josephine Nefkens Institute, Erasmus University Rotterdam, the Netherlands) and Mr. Steven Gheyselinck (Bibliothèque Centrale, École Polytechnique Fédérale de Lausanne, Switzerland) for providing them with copies of references [4] and [3], respectively. They would also like to thank the secondly assigned Associate Editor and reviewers for their contributions in bringing the review of this paper, as historical as the paper's subject matter, to a happy ending. 


\section{References}

[1] E. Catmull \& R. Rom, "A Class of Local Interpolating Splines", in Computer Aided Geometric Design, R. E. Barnhill \& R. F. Riesenfeld (eds.), Academic Press, New York, NY, 1974, pp. $317-326$.

[2] T. N. E. Greville, "The General Theory of Osculatory Interpolation", Transactions of the Actuarial Society of America, vol. 45, 1944, pp. 202-265.

[3] R. Henderson, "A Practical Interpolation Formula. With a Theoretical Introduction", Transactions of the Actuarial Society of America, vol. 9, no. 35, 1906, pp. 211-224.

[4] J. Karup, "Über eine Neue Mechanische Ausgleichungsmethode", in Transactions of the Second International Actuarial Congress, G. King (ed.), Charles and Edwin Layton, London, 1899, pp. 31-77. Translation: "On a New Mechanical Method of Graduation", pp. 78-109.

[5] R. G. Keys, "Cubic Convolution Interpolation for Digital Image Processing", IEEE Transactions on Acoustics, Speech, and Signal Processing, vol. 29, no. 6, 1981, pp. 1153-1160.

[6] G. King, "Notes on Summation Formulas of Graduation, with Certain New Formulas for Consideration", Journal of the Institute of Actuaries, vol. 41, 1907, pp. 530-565.

[7] T. M. Lehmann, C. Gönner, K. Spitzer, "Survey: Interpolation Methods in Medical Image Processing", IEEE Transactions on Medical Imaging, vol. 18, no. 11, 1999, pp. 1049-1075.

[8] E. H. W. Meijering, W. J. Niessen, M. A. Viergever, "Quantitative Evaluation of ConvolutionBased Methods for Medical Image Interpolation", Medical Image Analysis, vol. 5, no. 2, 2001, pp. 111-126.

[9] S. S. Rifman, "Digital Rectification of ERTS Multispectral Imagery", in Proceedings of the Symposium on Significant Results Obtained from the Earth Resources Technology Satellite-1, vol. 1, section B, NASA SP-327, 1973, pp. 1131-1142.

[10] R. W. Schafer \& L. R. Rabiner, "A Digital Signal Processing Approach to Interpolation", Proceedings of the IEEE, vol. 61, no. 6, 1973, pp. 692-702.

[11] A. Schaum, "Theory and Design of Local Interpolators", CVGIP: Graphical Models and Image Processing, vol. 55, no. 6, 1993, pp. 464-481.

[12] I. J. Schoenberg, "Contributions to the Problem of Approximation of Equidistant Data by Analytic Functions", Quarterly of Applied Mathematics, vol. 4, no. 1 \& 2, 1946, pp. 45-99 \& $112-141$.

[13] E. S. W. Shiu, "A Survey of Graduation Theory", in Actuarial Mathematics, H. H. Panjer (ed.), vol. 35 of Proceedings of Symposia in Applied Mathematics, American Mathematical Society, Providence, RI, 1986, pp. 67-84.

[14] P. Thévenaz, T. Blu, M. Unser, "Interpolation Revisited", IEEE Transactions on Medical Imaging, vol. 19, no. 7, 2000, pp. 739-758. 